\title{
Achieving Universal Access for Human Immunodeficiency Virus and Tuberculosis: Potential Prevention Impact of an Integrated Multi-Disease Prevention Campaign in Kenya
}

\author{
Reuben Granich, ${ }^{1}$ Nicolas Muraguri, ${ }^{2}$ Alexandre Doyen, ${ }^{3}$ \\ Navneet Garg, ${ }^{4}$ and Brian G. Williams ${ }^{5}$ \\ ${ }^{1}$ Treatment and Care Unit, Department of HIV/AIDS, World Health Organization, Avenue Appia 20, \\ 1211 Geneva, Switzerland \\ ${ }^{2}$ Ministry of Public Health and Sanitation, Government of Kenya, Nairobi, Kenya \\ ${ }^{3}$ Vestergaard Frandsen (EA) Ltd., Waiyaki Way, ABC Place, P.O. Box 66889, Nairobi 00800, Kenya \\ ${ }^{4}$ Innovation Centre, Vestergaard Frandsen, Chemin de Messidor 5-7, 1006 Lausanne, Switzerland \\ ${ }^{5}$ South African Centre for Epidemiological Modeling and Analysis, Stellenbosch, South Africa
}

Correspondence should be addressed to Reuben Granich, granichr@who.int

Received 30 November 2011; Revised 27 January 2012; Accepted 28 January 2012

Academic Editor: Anthony Harries

Copyright (c) 2012 Reuben Granich et al. This is an open access article distributed under the Creative Commons Attribution License, which permits unrestricted use, distribution, and reproduction in any medium, provided the original work is properly cited.

\begin{abstract}
In 2009, Government of Kenya with key stakeholders implemented an integrated multi-disease prevention campaign for waterborne diseases, malaria and HIV in Kisii District, Nyanza Province. The three day campaign, targeting 5000 people, included testing and counseling (HTC), condoms, long-lasting insecticide-treated bednets, and water filters. People with HIV were offered on-site CD4 cell counts, condoms, co-trimoxazole, and HIV clinic referral. We analysed the CD4 distributions from a district hospital cohort, campaign participants and from the 2007 Kenya Aids Indicator Survey (KAIS). Of the 5198 individuals participating in the campaign, all received HTC, 329 (6.3\%) tested positive, and $255(5 \%)$ were newly diagnosed (median CD4 cell count 536 cells/ $\mu \mathrm{L}$ ). The hospital cohort and KAIS results included 1,284 initial CD4 counts (median 348/L) and 306 initial CD4 counts (median $550 / \mu \mathrm{L}$ ), respectively (campaign and KAIS CD4 distributions $P=0.346$; hospital cohort distribution was lower $P<0.001$ and $P<0.001$ ). A Nyanza Province campaign strategy including ART $<350$ CD4 cell count could avert approximately 35,000 HIV infections and 1,240 TB cases annually. Community-based integrated public health campaigns could be a potential solution to reach universal access and Millennium Development Goals.
\end{abstract}

\section{Introduction}

In 30 years since the start of the human immunodeficiency virus (HIV) pandemic over 25 million people have died $[1,2]$. In 2010, an estimated 34 million people were living with HIV and $67 \%$ of them lived in sub-Saharan Africa [3]. Antiretroviral therapy (ART) has considerable potential to save lives while reducing the HIV transmission [4-7]. By the end of 2010, 6.6 million people were on antiretroviral treatment (ART) in the world [3]. Despite this remarkable achievement, an estimated 7.5 million people with CD4 cell counts $<350 / \mu \mathrm{L}$ were still in need of treatment [3]. Without a dramatic reduction in HIV incidence it is unlikely that we will be able to meet the growing demand for ART $[3,8]$. Addressing this prevention gap will require innovative approaches to improving access to HIV services including HIV testing and counselling (HTC) and ART.

Community-based efforts, including outreach beyond health facilities, may provide one approach to help bridge this gap. Of the 34 million people living with HIV, a majority are still unaware of their HIV status [3]. WHO, recognizing the need to markedly scale-up access to HTC, has recommended provider initiated HIV testing and counselling [9]. The Kenya National HIV and AIDS Strategic Plan III includes an HTC target of 18 million (80\%) of people $15-49$ years of age to be newly tested by 2013; however, despite increases 
in facility-based HTC the 2007 Kenya Aids Indicator Survey (KAIS) found that only $36 \%$ of adults have ever had an HIV test, and less than $20 \%$ of HIV-infected adults know that they are infected $[10,11]$. Obstacles to access to HTC include a shortage of trained counsellors, limited services, high transportation costs, limited test kit availability, and stigma [10-15]. Home-based HTC offers an important potential strategy to reach targets by expanding access beyond health care facilities. With a reported uptake of up to $90 \%$ in some settings, home-based HTC also provides an opportunity for couples counselling and mutual disclosure [15]. In Uganda's Bushenyi District, a 2.5 year multi-disease house-to-house HIV testing and counselling programme tested 264,996 (94\% acceptance) people in 92,984 (63\%) households [14].

Although home-based service delivery is feasible in many settings, it can be time and labour-intensive. Complementary community-based health campaigns are well suited to delivering services to the rural poor and have been used to deliver HIV counselling and testing and other simple interventions to large populations $[16,17]$. By combining multiple interventions and placing a larger proportion of the transaction costs onto the provider, integrated multi-disease campaigns create efficiencies for both the consumer and provider [16-19]. There is considerable experience with the health campaign approach including the mass distribution of insecticide-treated bednets which have quickly reached high coverage levels at low cost and are associated with declines in child and adult mortality in east Africa [16-19]. More recent work in sub-Saharan Africa has focused on bundling multiple interventions into a multi-disease prevention package which includes long-lasting impregnated bednets, water purification systems, preventive health education, condoms, and cotrimoxazole prophylaxis for HIV-infected adults [16, 17, 20-22].

Building on the previous community-based campaign experience in Kenya's Kakamega District, in 2009 we implemented a similar multi-disease prevention (MDP) campaign in Kisii District, Nyanza Province [16]. We applied lessons learned to pilot streamlined HTC protocols, provide sameday, onsite access to CD4 cell counts, and strengthen linkage to care. Our study examines the HIV component of the Kisii District campaign and compares CD4 count distributions to explore whether a multi-disease campaign strategy can improve earlier access to HIV diagnosis and treatment. We also project the potential HIV and TB prevention impact achieved by reaching people earlier for different ART eligibility scenarios.

\section{Methods}

2.1. Multi-Disease Prevention Campaign. In 2008 the Ministry of Health Kenya, the United States Government Centers for Disease Control Kenya, Community Housing Foundation (a local NGO), and Vestergaard Frandsen (a private sector manufacturing company focused on products that address the MDGs) implemented a 7 day multi-disease prevention campaign that reached 47,311 (92\%) of adults 15-49 years old in Kakamega District, Western Province of Kenya. The campaign was in line with the Kenyan National AIDS
Strategic Plan III's target to reach $80 \%$ of $15-64$ year olds and provided interventions to address HIV, malaria, and diarrhea $[10,16]$. Point-of-care CD4 counts were also piloted in selected sites [16]. Nyanza Province in western Kenya, has a high incidence of malaria, diarrhoeal disease, and tuberculosis $[3,10,11,23-25]$. In 2007, an estimated $15 \%$ of $15-$ 64 year olds were living with HIV [11] which has contributed to the high annual $\mathrm{TB}$ incidence (353 per 100,000 population) $[11,24,25]$. Standard operating procedures from the Kakamega campaign [16] were modified for the Kisii District Campaign as described below.

In September 2009 we implemented a three-day multidisease prevention campaign that targeted diarrhoeal diseases, malaria and HIV in Kisii District, Nyanza Province (population 4,392,000). Three peri-urban campaign sites were set up around health facilities in the periphery of Kisii (Figure 1 map). The campaign was designed to (1) apply lessons learned from previous campaigns to a peri-urban setting, (2) pilot improved HIV testing and counselling protocols, (3) strengthen the referral system for improved linkage to care, (4) determine the feasibility of providing same day CD4 cell count testing for all HIV-positive participants and, (5) explore the potential impact of campaigns for early identification for HIV prevention and care services, and (6) examine differences between campaign, hospital, and provincial populations.

The campaign was planned to provide services for 5000 adults within 3 days by using a specific protocol adapted to mass campaign settings. We conducted the campaign over a weekend (Saturday, Sunday, and Monday) to ensure maximum participation of both men and women and limit disruptions to routine services. A pre-campaign social mobilization exercise started one month before the start date and engaged the community using village "baraza" forums with local chiefs, radio and print messaging, and town cries with mobile trucks. Participants were informed about campaign services, campaign sites and provided health education messages around diarrhea, malaria, HIV, and STDs. They were offered services on a first-come first-serve basis and census lists from 2008, identification cards, and indelible finger print dye were used to ensure that participants could only participate once in the campaign (all participants received finger dye irrespective of the services accessed or serostatus). To ensure that people accessing services were from the immediate local area, we used village elders and government officers working at the village level to advertise the campaign and carefully recorded location information during the registration process.

A total of 90 counselors were hired and trained to use the mass testing protocol designed for community-based campaigns with a target of 25 clients per day. Pretest counselling was offered by trained Health Communication Officers to groups of 20 participants selected for age and gender as they waited to meet the HTC counsellors. Confidentiality, consent, and counselling were assured by issuance of cards with a unique identifier number during the registration process and it was emphasized to all participants that HTC was entirely voluntary and that everyone would receive the other interventions whether or not they opted for HIV 


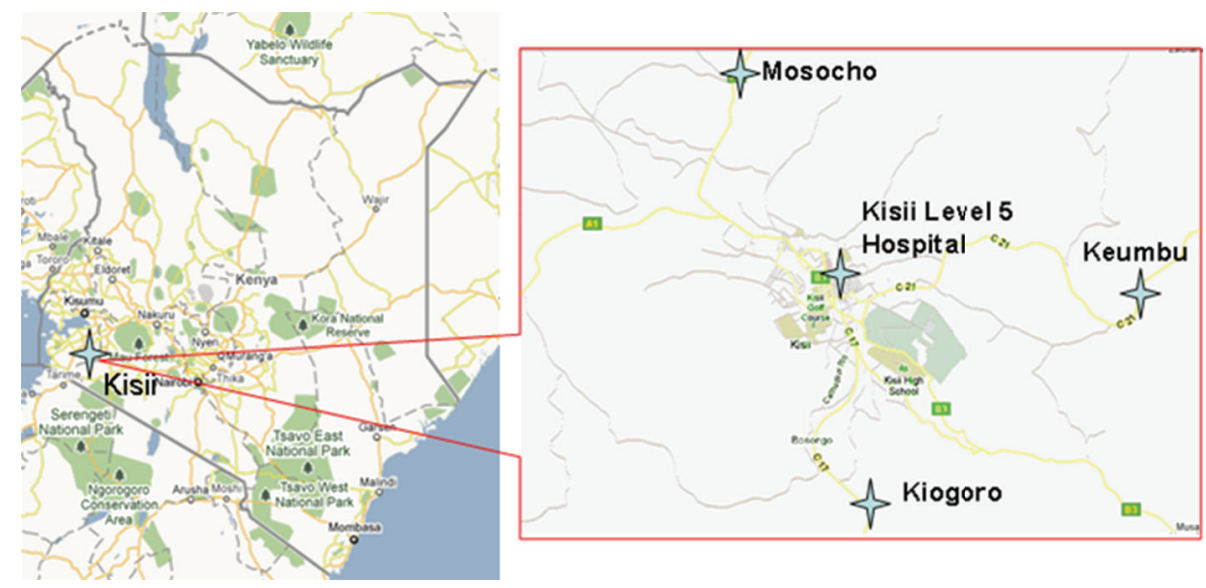

FIgure 1: Map of Kenya showing Kisii, Nyanza Province, and inset showing the location of the three campaign sites and Kisii Level 5 Hospital (Kisii Town).

testing. HTC was provided on an "opt-in" basis and written consent was required for testing. Quality of HTC was insured by the use of certified counsellors, refresher training courses, a supervision system which employed one supervisor for every 10 counsellors, sending 1 out of 50 blood samples for reconfirmation with a different diagnostic method (PCR), and exit interviews by trained staff for all campaign participants. The exit interviews were used to assure quality and to improve services on a real-time basis.

Participants who tested HIV positive received a 3month supply of cotrimoxazole, same-day on-site CD4 count measurement, psychosocial support, local referral for further care, and were offered enrolment in a support network by peer counsellors. Linkage to care was given a high priority and planned for through various interventions. Counselors emphasized the importance of care during post-test counselling. Members from local people living with HIV (PLWHA) support networks were enrolled and trained for the implementation of the PLWHA navigator approach. As part of the navigator strategy, people testing HIV positive were offered further counselling by assigned PLWHA counsellors and, with consent, were enrolled into local support groups. Most participants opted to allow follow-up visits and provided name, address, unique identifier number, and phone number. PLWHA counsellors, using a list of clients, checked in with health centers on a monthly basis and, if necessary, made follow-up household visits [26].

The Kisii campaign included provision of one long-lasting insecticide-treated net per participant, water filters (individual filter for men, household filter for women), 60 condoms per person, and health education encompassing HIV, sexually transmitted infections, malaria, and water-borne diseases. The unit cost per person by disease was $\$ 6.27$ for malaria (nets and training), $\$ 15.80$ for diarrhea (filters and training), and \$9.91 for HIV (test kits, counselling, condoms, and CD4 testing) [16, 27]. Using logistic and expenditure data from the 2008 Lurambi District Campaign, [16] the cost of a scaled-up replication (SUR) was estimated assuming reliance on local managers, potential efficiencies of scale, and other adjustments (Jim Kahn, personal communication).
The SUR cost of $\$ 31.98$ per person included $67 \%$ for commodities (mainly water filters and bed nets) and $20 \%$ for personnel.

\subsection{Measurement and Analysis of CD4 Cell Count Distribu-} tions. Absolute CD4 cell counts and total lymphocyte counts were performed by portable Guava AUTOCD4 flow cytometers manufactured by Millipore. All three sites were equipped with a unit with a single 150 amperes battery ( 7 hour off-grid capacity). Samples were processed in batches and had a 45minute incubation time and 4-minute processing time. Each unit had a trained machine operator and a trained nurse or other health care provider responsible for drawing $10 \mu \mathrm{L}$ of whole blood (EDTA) and preparing samples for analysis. As patients waited for their results, they were given additional psychosocial counselling by a counsellor living with HIV. For external quality control, 5 percent of all blood samples were sent for confirmation at Kisii Level 5 Hospital Laboratory using a Becton Dickinson Facs Calibur Flow Cytometer. The Kisii Hospital laboratory routinely sends $10 \%$ of blood samples to CDC Kisumu for external quality control.

To create a matching historical cohort and a baseline for comparison, we abstracted the medical records, including the first measured CD4 count, for all newly diagnosed patients aged 15 and above from March to August 2009 at the HIV/AIDS Patient Support Center in the Kisi District Level 5 Hospital (apex of district health care facilities). All CD4 measurements for this cohort were made using the same Kisii Level 5 Hospital laboratory Becton Dickinson Facs Calibur Flow Cytometer.

We analyzed the CD4 data from the Kisii campaign, the reference hospital and Nyanza province data from the recently performed KAIS survey [11]. The 2007 KAIS is the first national population-based survey of Kenya that obtained representative estimates on behavioral, clinical and biologic indicators for HIV/AIDS. The 2007 KAIS was conducted among a sample of households selected from all eight provinces in the country, covering both rural and urban areas (more detailed methods are described in detail elsewhere) [11]. 


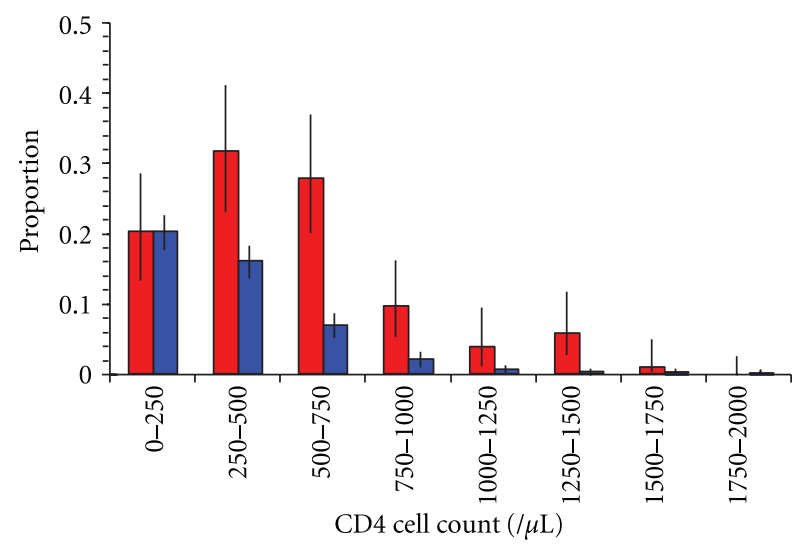

FIgure 2: Comparison of the CD4 cell count distribution in Nyanza Province (red; KAIS survey) and the Kisii Hospital cohort (blue). The data for the hospital cohort are scaled to match the KAIS data for the lowest CD4 cell count range and the differences in the heights of the bars for the higher ranges show the proportion that are missed in the hospital cohort.

\subsection{Projecting the Potential HIV and TB Prevention Impact} of Early Detection. For our comparison and projections we used data from those who were reported as being newly diagnosed-all were assumed to be ART naive. Cumulative distribution functions were compared using the standard Kolmogorov-Smirnov test. We estimated the potential benefits of early identification and starting ART for three scenarios (1) <250 CD4 cells (status quo), (2) <350 CD4 cells, and (3) immediate ART irrespective of CD4 cell count.

To estimate the proportion of people with CD4 cell counts that are missed under passive clinic-based case-finding but would be found using a campaign approach, we assumed that everyone with a CD4 cell count below $250 / \mu \mathrm{L}$ will present to a health facility before they die. We scaled the proportions in the "hospital reference" data so as to match the proportion in the KAIS data set below $250 / \mu \mathrm{L}$ (Figure 2). Applying the scaling proportion allows us to see the differences in proportions of people that are missing in the hospital cohort at the higher CD4 levels. This in turn enables us to obtain an approximate estimate of the increase in the number of people who would be put onto ART and the number of TB cases that would be averted by adopting a campaign approach.

The population of Nyanza province is 4.4 million of whom 2.9 million are adults with around 435,000 (15\%) who are estimated to be HIV positive [11]. We estimated the number of HIV-positive TB patients in Nyanza Province in two ways. First, the case-notification rate of HIV-positive TB patients in Nyanza is 189 per 100,000 population giving about $8,314 \mathrm{HIV} / \mathrm{TB}$ cases per year [25]. Second, the life-time risk of TB for those not receiving ART has been estimated to be $13 \%$ [28]. With a mean life expectancy of HIV-positive people of ten years, this means that the annual risk of TBdisease is $1.3 \%$ and we expect there to be about 435,000 $\times 0.013=5655$ case of TB in HIV-positive people in the province each year. Taking the average of these two estimates, the expected number is 6,985 per year. ART reduces the incidence of TB by about 71\% [28], so that if everyone started ART immediately they were found to be HIV-positive the number of TB cases averted would be 4,959. This enables us to estimate the reduction in the number of $\mathrm{TB}$ cases that we expect each year under the campaign and passive clinic-based case-finding approaches.

To estimate the number of new HIV transmissions averted, we assumed that the epidemic is in a steady state so that each person with HIV infects one other person before they die. Assuming that the CD4 cell count is $750 / \mu \mathrm{L}$ immediately after seroconversion [29] we multiply the number started on ART by the proportion of time for which they are on ART (the CD4 cell count at the time at diagnosis in the different scenarios is used to calculate the amount of time on and off ART). To derive the HIV infections averted we compared the projected outcome using these assumptions with "no ART." To simplify the analysis, we did not include the additional prevention benefits of the long-term reduction in TB transmission and treatment of identified TB cases. We also did not factor in WHO recommended IPT or infection control for TB which is not yet in widespread use in Kenya [30].

The study protocol was reviewed by the Kenya Ministry of Public Health and Sanitation and considered to be part of on-going program monitoring and evaluation. The study represented a private-public partnership and funding for the study was provided by the Kenya Ministry of Public Health as part of routine public health services. Ministry of Health Kenya and Vestergaard Frandsen funded the campaign; $\mathrm{MOH}$ provided campaign personnel, HIV test kits, and condoms. The decision to conduct, analyse, and submit the study was taken by the Ministry of Health and WHO.

\section{Results}

3.1. Multi-Disease Prevention Campaign. Over a three-day period, the campaign reached 5198 individuals aged over 15 years with a $100 \%$ uptake of the HIV counselling and testing and multi-disease preventive package. Counselors worked 8 hour days starting from 8:00 AM and tested around 25 clients per day (100\% of target). Clients who were found to be HIV negative were provided HTC in about 20 minutes, while those who were diagnosed with HIV were given HTC counseling in about 38 minutes. The process from drawing blood to getting CD4 cell count results usually took around two hours (mean 119; range 47-191 minutes).

Of the 5198, 2090 (40\%) were males. Of the 329 participants who tested HIV positive, 71 (22\%) were males; HIV prevalence among males was $3.3 \%$ and $8.3 \%$ for females. This difference of HIV prevalence between genders reflects the 2010 antenatal care sentinel surveillance results of $8.7 \%$ among women in Kisii District sites [31]. A separate study that included a subsample of the people from the Kisii campaign and others evaluated factors affecting linkage to care and found that $81 \%$ of people who consented to followup visited the referral clinic by 10 months after the campaign [26]. 
TABLE 1: CD 4 values from the campaign, hospital reference, and KAIS data sets. The table gives $N$, the number of people for whom a CD4 cell count was done, the median CD4 cell count, and the proportion of those tested that are below 250, 350, and 500 cells $/ \mu \mathrm{L}$. Numbers in brackets are percentages. Using a Kolmogorov-Smirnov test, The CD4 cell count distribution for the Hospital Reference data set is significantly different from the other two $(P<0.001$ in both cases) but the Campaign and KAIS data sets are not significantly different $(P=0.346)$.

\begin{tabular}{lccc}
\hline & Campaign & Hospital reference & Nyanza KAIS \\
\hline$N$ & 255 & 1284 & 306 \\
\hline Median/ $\mu \mathrm{L}$ & 536 & 348 & 550 \\
$N<250$ & $33(13 \%)$ & $436(34 \%)$ & $52(17 \%)$ \\
$N<350$ & $64(25 \%)$ & $642(50 \%)$ & $92(30 \%)$ \\
$N<500$ & $112(44 \%)$ & $899(70 \%)$ & $141(46 \%)$ \\
\hline$N<750$ & $187(74 \%)$ & $1137(89 \%)$ & $220(72 \%)$ \\
\hline$N<1000$ & $228(90 \%)$ & $1215(95 \%)$ & $258(84 \%)$ \\
\hline
\end{tabular}

3.2. Analysis of CD4 Cell Count Distributions. Of the 258 (4.9\%) who were newly diagnosed with HIV (71 knew their status before campaign), CD4 count determination was performed for 255 (98\%). The median CD4 count was 536 cells/ $\mu \mathrm{L}$ (IQR 348 to 760 ;) with 13\% having a CD4 count $<250$ cell $/ \mu \mathrm{L}$ and $25 \%$ a CD 4 cell count $<350$ cells $/ \mu \mathrm{L}$ (Table 1).

Of the 1284 patients in the Kisii Hospital reference cohort, 350 (27\%) were male (age range 15-61; CD4 count range 1-1862) and $934(73 \%)$ were female (age range 15-69; CD4 count range 1-2560). The first CD4 counts from the 1284 patients had a median of 348 (IQR 185 to 551) with $34 \%$ having a CD 4 count $<250$ cell $/ \mu \mathrm{L}$ and $50 \%$ a CD 4 cell count $<350$ cells $/ \mu \mathrm{L}$ (Table 1 ).

The results obtained from the 2007 KAIS data base for Nyanza Province included 1585 females, 1386 (87\%) tested, 240 (17\%) HIV positive, 218 (91\%) not on ART, and 203 (85\%) with CD4 counts. Of the 1229 males surveyed, 994 (81\%) were tested, 123 (12\%) HIV positive, 108 (88\%) not on ART, and 103 (95\%) with CD4 counts. The median CD4 count overall was 550 cells/ $\mu \mathrm{L}$ (IQR 305 to 785). Table 1 shows that the CD4 cell count data from the campaign for Kisii are not significantly different from the KAIS data for Nyanza $(P=0.346)$.

\subsection{Projecting HIV and TB Prevention Impact of Early Detec-} tion. Figure 2 shows that the Hospital reference cohort has significantly lower median CD4 cell counts when compared with the Campaign and KAIS data. Table 2 shows that using our scaled estimation approach with either campaign or hospital-based strategies, current $\leq 250$ ART eligibility criteria results in around 38,000 people started on ART and about 645 cases of TB will be averted in Nyanza Province. Increasing the CD4 cell count eligibility to $\leq 350$ combined with the passive case-finding approach increases the number starting ART to 56,000, averts 26,000 new HIV infections, and prevents 942 TB cases. However, the $\leq 350$ ART eligibility criteria combined with the campaign approach would translate into an estimated 74,000 people starting ART, thereby averting 35,000 new HIV infections and preventing 1,240 TB cases per year. Starting at a CD4 cell count of $500 / \mu \mathrm{L}$ gives an even greater relative advantage to the campaign approach with 129,000 , or 2.6 times as many people started on ART, and 2,182 total or 2.6 times as many TB cases averted using the campaign approach when compared with the passive case-finding approach.

\section{Discussion}

This three-day integrated multi-disease prevention campaign reached over five thousand people in Kisii district including over 200 people who were unaware that they were living with HIV. The uptake of HCT in the campaign is comparable to the high rates of over $90 \%$ observed in home-based, door-to-door testing interventions implemented in Uganda $[15,32,33]$ and Kenya [34] and was achieved in considerably less time. Similar to previous campaigns, [16] successful implementation of this campaign may have been due to the engagement of the community leadership, delivery of services at convenient locations near participants' homes and the multi-disease prevention approach which included concomitant distribution of free nets, water filters, and condoms. Although access to laboratory tests including CD4 levels has been a major barrier to expanding access to ART $[35,36]$, the campaign successfully delivered same-day CD4 level testing results for all of the newly identified people with HIV.

Delayed diagnosis and access to ART have significant public health implications for both the individual and the community. Expanded access to HCT linked with point-ofcare CD4 testing has considerable potential to support the implementation of WHO's recommendation to start ART for everyone with a CD4 $\leq 350 / \mu \mathrm{L}$ [37]. Comparison of CD4 counts from campaign participants with the hospital cohort CD4 data and the recent national survey suggests that the campaign identified people significantly earlier in the course of their HIV disease. This makes intuitive sense as it reaches people before they are symptomatic and is supported by other studies examining the use of community-based services outside health facilities [38]. Although we do not present the data, the $80 \%$ linkage to care for people diagnosed with HIV in this campaign at 10 months was better than in many other settings [39] but required setting up a robust follow-up system. The data also suggest that increasing the threshold to $350 / \mu \mathrm{L}$ combined with the standard passive facility-based case-finding approach could increase the number of people in Nyanza who need to start ART by a factor of 1.9 or 56,000 people. However, the campaign approach combined with optimal linkage to care could increase the number receiving ART by a factor of 3.7 or 74,000 additional people-an additional 18,000 people who were unaware of their HIV status and who were eligible but not on ART. Our simple projections using a stable generalized epidemic setting suggest that an active campaign approach to identify those with CD4 cell count $<350$ could prevent 10,000 HIV transmissions, 76,000 deaths and $3600 \mathrm{~TB}$ cases per year. Although more complex projections for the province and country are beyond the scope of this paper, improving access to early 
TABle 2: Projected prevention impact of campaign approach by CD4 eligibility criteria for Nyanza Province.

\begin{tabular}{|c|c|c|c|c|c|c|c|c|}
\hline \multirow[b]{2}{*}{$\begin{array}{l}\text { CD4 cell count } \\
\text { at start of } \\
\text { treatment }(/ \mu \mathrm{L})\end{array}$} & \multicolumn{4}{|c|}{ Campaign approach } & \multicolumn{4}{|c|}{ Passive case-finding } \\
\hline & $\begin{array}{l}\text { HIV-positive } \\
\text { population } \\
\text { started on ART } \\
(\%)\end{array}$ & $\begin{array}{c}\text { Number started } \\
\text { on ART } \\
\text { (thousands) }\end{array}$ & $\begin{array}{l}\text { HIV infections } \\
\text { averted per year } \\
\text { (thousands) }\end{array}$ & $\begin{array}{l}\text { TB cases } \\
\text { averted per } \\
\quad \text { year }\end{array}$ & $\begin{array}{l}\text { HIV- } \\
\text { positive } \\
\text { population } \\
\text { started on } \\
\text { ART }(\%)\end{array}$ & $\begin{array}{c}\text { Number } \\
\text { started on } \\
\text { ART } \\
\text { (thousands) }\end{array}$ & $\begin{array}{c}\text { HIV } \\
\text { infections } \\
\text { averted per } \\
\text { year } \\
\text { (thousands) }\end{array}$ & $\begin{array}{c}\text { TB cases } \\
\text { averted per } \\
\text { year }\end{array}$ \\
\hline$\leq 250$ & 13 & 38 & 13 & 645 & 13 & 38 & 13 & 645 \\
\hline$\leq 350$ & 25 & 74 & 35 & 1240 & 19 & 56 & 26 & 942 \\
\hline$\leq 500$ & 44 & 129 & 86 & 2182 & 27 & 79 & 53 & 1339 \\
\hline Immediate & 100 & 294 & 294 & 4959 & 38 & 112 & 112 & 1884 \\
\hline
\end{tabular}

ART through a campaign approach could have significant public health and economic benefits including preventing morbidity, mortality, disease transmission, and reducing costs to the individual, health system, and society $[4,6,40]$.

Short intense multi-disease campaigns face a number of challenges including maintaining efficiency and quality of service provision and linkage to care while dealing with large numbers of people. Previous work in Kenya and elsewhere suggests that careful consensus building and micro-planning with community leaders and key health care providers is required to mobilize resources and provide high-quality services for the temporary surge of participants in the campaign [16]. The various TB and HIV prevention scenarios modeled would only be achievable under conditions of a high linkage to care after the campaign which requires postcampaign systems monitoring health care facility attendance, active followup, and local support networks. Another significant challenge is the cost of the campaign. Preliminary analyses suggest that despite the relative high costs per person [27] the campaign is likely to be cost effective in part due to the multi-disease approach and the numbers of people reached in a short period of time. Arguably, delivering health care services from fixed facilities is also costly and often does not reach stated objectives.

There are important limitations to our study. The comparison of the hospital, province, and campaign CD4 data may have been influenced by a number of biases introduced from the selection of the three populations. Specifically, it is difficult to say with certainty that the three subpopulations that we compared are similar given the different ways that people accessed the hospital, campaign and KAIS survey (e.g., nonresponse, refusal, and missing CD4 counts). Additionally, there are potential confounders that may have affected the CD4 results including the difference in methods to determine CD4 counts, diurnal rhythms, physical and psychological stress, pregnancy, drug administration, tuberculosis, and viral infections. However, the similarity of the campaign data with the provincial data for Nyanza is reassuring and the lower CD4 counts of those who are ill and seeking care in a hospital setting make sense. Our assumption that people coming into the hospital for care were not referred from a peripheral site and the high linkage to care may have resulted in optimistic projections favoring the campaign strategy. However, despite our lack of certainty regarding the projected benefits which relied on crude estimates, we are likely to be directionally correct and a more sophisticated modeling approach may provide additional insights.

We are far from achieving universal access and there is increasing interest in new approaches to ensuring early and equitable access to ART and other HIV services. This multidisease prevention campaign presents an operational proof of concept for the expanded access to HTC and same-day CD4 testing that is required for many countries to reach national HIV and TB prevention goals. Multi-disease integrated campaigns have considerable potential and may represent an important conceptual breakthrough in our efforts to achieve national health objectives reflected in the Millennium Development Goals [41].

\section{Authors' Contribution}

R. Granich is the Lead Author. R. Granich, N. Muraguri, A. Doyen, N. Garg, and B. Williams made the study design; N. Muraguri, A. Doyen, and N. Garg were responsible for the collection of data; B. Williams, A. Doyen, and R. Granich made the analysis; R. Granich, N. Muraguri, A. Doyen, N. Muraguri, and B. Williams were responsible for interpretation of data; R. Granich, N. Muraguri, A. Doyen, N. Garg, and B. Williams made the draft paper; R. Granich, N. Muraguri, A. Doyen, N. Muraguri, and B. Williams prepared the final paper.

\section{Disclaimer}

The opinions and statements in this paper are those of the authors and do not represent the official policy, endorsement, or views of the World Health Organization.

\section{Conflict of Interests}

None of the authors has conflict of interests to declare.

\section{References}

[1] F. Barre Sinoussi, J. C. Chermann, and F. Rey, "Isolation of a Tlymphotropic retrovirus from a patient at risk for acquired immune deficiency syndrome (AIDS)," Science, vol. 220, no. 4599, pp. 868-871, 1983. 
[2] C. W. Dieffenbach and A. S. Fauci, "Thirty years of HIV and AIDS: future challenges and opportunities," Annals of Internal Medicine, vol. 154, no. 11, pp. 766-771, 2011.

[3] WHO, "Towards Universal Access: scaling up priority HIV/AIDS interventions in the health sector," 2011, http:// whqlibdoc.who.int/publications/2011/9789241502986_eng .pdf.

[4] R. M. Granich, C. F. Gilks, C. Dye, K. M. De Cock, and B. G. Williams, "Universal voluntary HIV testing with immediate antiretroviral therapy as a strategy for elimination of HIV transmission: a mathematical model," The Lancet, vol. 373, no. 9657, pp. 48-57, 2009.

[5] D. Donnell, J. M. Baeten, J. Kiarie et al., "Heterosexual HIV-1 transmission after initiation of antiretroviral therapy: a prospective cohort analysis," The Lancet, vol. 375, no. 9731, pp. 2092-2098, 2010.

[6] J. S. Montaner, R. Hogg, E. Wood et al., "The case for expanding access to highly active antiretroviral therapy to curb the growth of the HIV epidemic," The Lancet, vol. 368, no. 9534, pp. 531-536, 2006.

[7] M. S. Cohen, Y. Q. Chen, M. McCauley et al., "Prevention of HIV-1 infection with early antiretroviral therapy," The New England Journal of Medicine, vol. 365, no. 6, pp. 493-505, 2011.

[8] B. Schwartländer, J. Stover, T. Hallett et al., "Towards an improved investment approach for an effective response to HIV/AIDS," The Lancet, vol. 377, no. 9782, pp. 2031-2041, 2011.

[9] WHO. Guidance on Provider-Initiated HIV Testing and Counseling in Health Facilities. WHO, Geneva, Switzerland, 2007, http://whqlibdoc.who.int/publications/2007/ 9789241595568_eng.pdf.

[10] Kenya National AIDS Strategic Plan (2009/10-2012/13): delivering on Universal Access to Services, 2009, http://www.hennet.or.ke/downloads/knasp_iii_document.pdf.

[11] National AIDS and STI Control Programme Ministry of Public Health and Sanitation-Kenya (2008) KAIS 2007, Kenya AIDS Indicator Survey Preliminary Report (KAIS). Nairobi, Kenya, 2007, http://www.nacc.or.ke/nacc\%20downloads/official_kais_report_2009.pdf.

[12] N. Menzies, B. Abang, R. Wanyenze et al., "The costs and effectiveness of four HIV counseling and testing strategies in Uganda," AIDS, vol. 23, no. 3, pp. 395-401, 2009.

[13] J. R. Kemp, G. Mann, B. N. Simwaka, F. M. L. Salaniponi, and S. B. Squire, "Can Malawi's poor afford free tuberculosis services? Patient and household costs associated with a tuberculosis diagnosis in Lilongwe," Bulletin of the World Health Organization, vol. 85, no. 8, pp. 580-585, 2007.

[14] E. Tumwesigye, G. Wana, S. Kasasa, E. Muganzi, and F. Nuwaha, "High uptake of home-based, district-wide, HIV counseling and testing in Uganda," AIDS Patient Care and STDs, vol. 24, no. 11, pp. 735-741, 2010.

[15] W. Were, J. Mermin, R. Bunnell, J. P. Ekwaru, and F. Kaharuza, "Home-based model for HIV voluntary counselling and testing," The Lancet, vol. 361, no. 9368, p. 1569, 2003.

[16] E. Lugada, D. Millar, J. Haskew et al., "Rapid implementation of an integrated large-scale hiv counseling and testing, malaria, and diarrhea prevention campaign in rural kenya," PLoS One, vol. 5, no. 8, Article ID e12435, 2010.

[17] M. Otten, M. Aregawi, W. Were et al., "Initial evidence of reduction of malaria cases and deaths in Rwanda and Ethiopia due to rapid scale-up of malaria prevention and treatment," Malaria Journal, vol. 8, no. 1, article 14, 2009.

[18] M. Grabowsky, N. Farrell, W. Hawley et al., "Integrating insecticide-treated bednets into a measles vaccination campaign achieves high, rapid and equitable coverage with direct and voucher-based methods," Tropical Medicine and International Health, vol. 10, no. 11, pp. 1151-1160, 2005.

[19] M. Grabowsky, T. Nobiya, M. Ahun et al., "Distributing insecticide-treated bednets during measles vaccination: a low-cost means of achieving high and equitable coverage," Bulletin of the World Health Organization, vol. 83, no. 3, pp. 195-201, 2005.

[20] J. R. Lule, J. Mermin, J. P. Ekwaru et al., "Effect of home-based water chlorination and safe storage on diarrhea among persons with human immunodeficiency virus in Uganda," American Journal of Tropical Medicine and Hygiene, vol. 73, no. 5, pp. 926-933, 2005.

[21] J. Mermin, J. P. Ekwaru, C. A. Liechty et al., "Effect of cotrimoxazole prophylaxis, antiretroviral therapy, and insecticide-treated bednets on the frequency of malaria in HIV-1infected adults in Uganda: a prospective cohort study," The Lancet, vol. 367, no. 9518, pp. 1256-1261, 2006.

[22] R. Colindres, J. Mermin, E. Ezati et al., "Utilization of a basic care and prevention package by HIV-infected persons in Uganda," AIDS Care, vol. 20, no. 2, pp. 139-145, 2008.

[23] R. Granich, S. Crowley, M. Vitoria et al., "Highly active antiretroviral treatment for the prevention of HIV transmission," Journal of the International AIDS Society, vol. 13, no. 1, article $1,2010$.

[24] WHO. Global tuberculosis control: epidemiology, strategy, financing WHO/HTM/TB/2009.411, 2009, http://www.who int/tb/publications/global_report/2009/pdf/full_report.pdf.

[25] WHO, A Brief History of Tuberculosis Control in Kenya, World Health Organization, Geneva, Switzerland, 2009.

[26] A. M. Hatcher, J. M. Turan, H. H. Leslie et al., "Predictors of linkage to care following community-based HIV counseling and testing in rural Kenya," AIDS and Behavior, p. 4, 2011.

[27] J. G. Kahn, B. Harris, J. H. Mermin et al., "Cost of community integrated prevention campaign for malaria, HIV, and diarrhea in rural Kenya," BMC Health Services Research, p. 6, 2011.

[28] B. G. Williams, R. Granich, K. M. De Cock, P. Glaziou, A. Sharma, and C. Dye, "Anti-retroviral therapy for the control of HIV-associated tuberculosis: modelling the potential effects in nine African countries," PNAS, vol. 11, article 346, 2011.

[29] B. G. Williams, E. L. Korenromp, E. Gouws, G. P. Schmid, B. Auvert, and C. Dye, "HIV infection, antiretroviral therapy, and $\mathrm{CD}^{+}$cell count distributions in African populations," Journal of Infectious Diseases, vol. 194, no. 10, pp. 1450-1458, 2006.

[30] WHO, Guidelines for Intensified Tuberculosis Case-Finding and Isoniazid Preventive Therapy for People Living with HIV in Resourceconstrained Settings, WHO, Geneva, Switzerland, 2011.

[31] National AIDS and STI Control Program. 2010. Sentinel surveillance for HIV and Syphilis among pregnant women. NASCOP, Nairobi, Kenya, 2010, http://www.nascop.or.ke/3d/.

[32] J. K. B. Matovu, G. Kigozi, F. Nalugoda, F. Wabwire-Mangen, and R. H. Gray, "The Rakai Project counselling programme experience," Tropical Medicine and International Health, vol. 7, no. 12, pp. 1064-1067, 2002.

[33] B. Wolff, B. Nyanzi, G. Katongole, D. Ssesanga, A. Ruberantwari, and J. Whitworth, "Evaluation of a home-based voluntary counselling and testing intervention in rural Uganda," Health Policy and Planning, vol. 20, no. 2, pp. 109-116, 2005.

[34] S. Kimaiyo, A. Siika, and P. Ayuo, "Effectiveness and outcomes of door-to-door HIV testing in a rural district of Western Kenya," in Proceedings of the 17th International AIDS Conference, Mexico City, Mexico, August 2008, Abstract no. TUPE0389. 
[35] G. M. Cohen, "Access to diagnostics in support of HIV/AIDS and tuberculosis treatment in developing countries," AIDS, vol. 21, supplement 4, pp. S81-S87, 2007.

[36] C. A. Petti, C. R. Polage, T. C. Quinn, A. R. Ronald, and M. A. Sande, "Laboratory medicine in Africa: a barrier to effective health care," Clinical Infectious Diseases, vol. 42, no. 3, pp. 377382, 2006.

[37] WHO, Rapid Advice: Antiretroviral Therapy for HIV Infection in Adults and Adolescents, WHO, Geneva, Switzerland, 2009.

[38] N. van Schaik, K. Kranzer, R. Wood, and L.-G. Bekker, "Earlier HIV diagnosis-are mobile services the answer?" South African Medical Journal, vol. 100, no. 10, pp. 671-674, 2010.

[39] S. Rosen and M. P. Fox, "Retention in HIV care between testing and treatment in sub-Saharan Africa: a systematic review," PLoS Medicine, vol. 8, no. 7, Article ID e1001056, 2011.

[40] E. Bendavid, M. L. Brandeau, R. Wood, and D. K. Owens, "Comparative effectiveness of HIV testing and treatment in highly endemic regions," Archives of Internal Medicine, vol. 170, no. 15, pp. 1347-1354, 2010.

[41] J. D. Sachs and J. W. McArthur, "The millennium project: a plan for meeting the millennium development goals," The Lancet, vol. 365, no. 9456, pp. 347-353, 2005. 


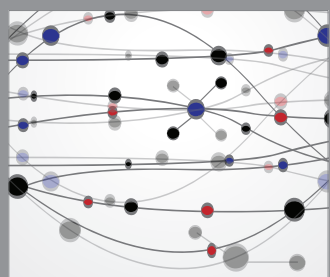

The Scientific World Journal
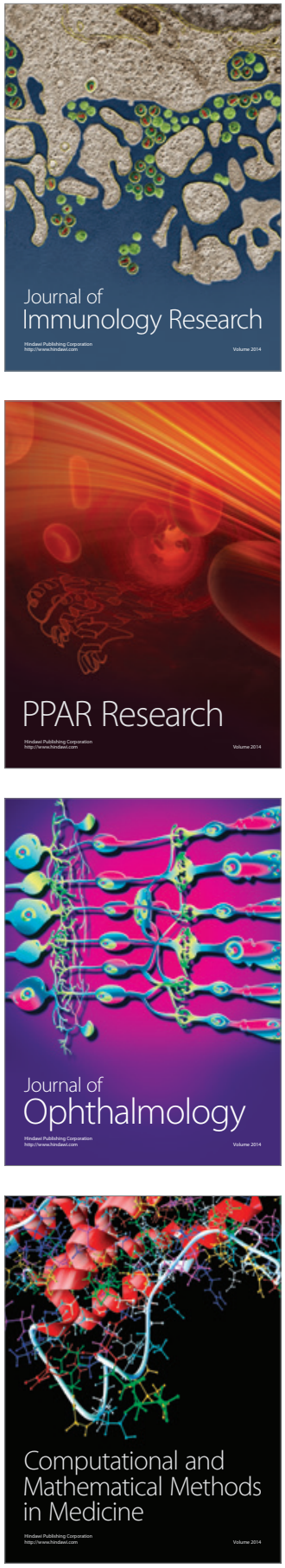

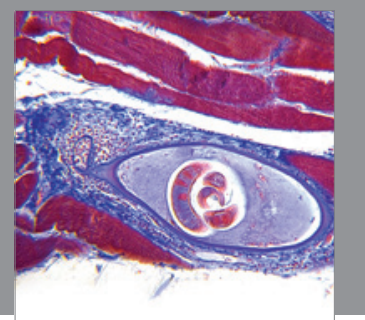

Gastroenterology

Research and Practice
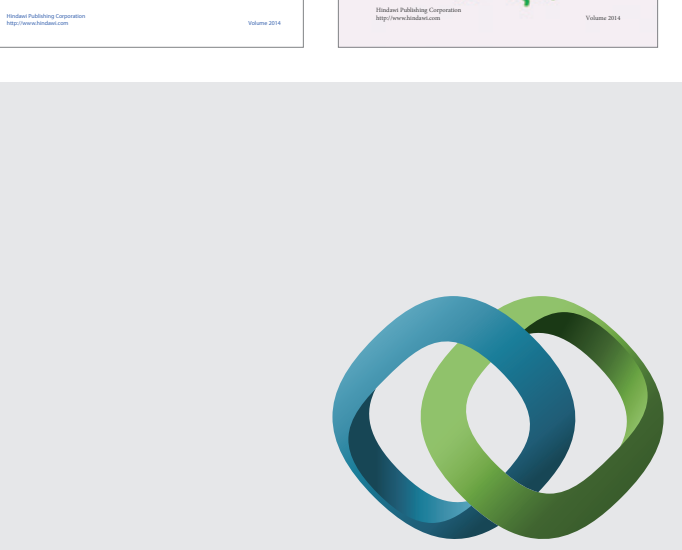

\section{Hindawi}

Submit your manuscripts at

http://www.hindawi.com
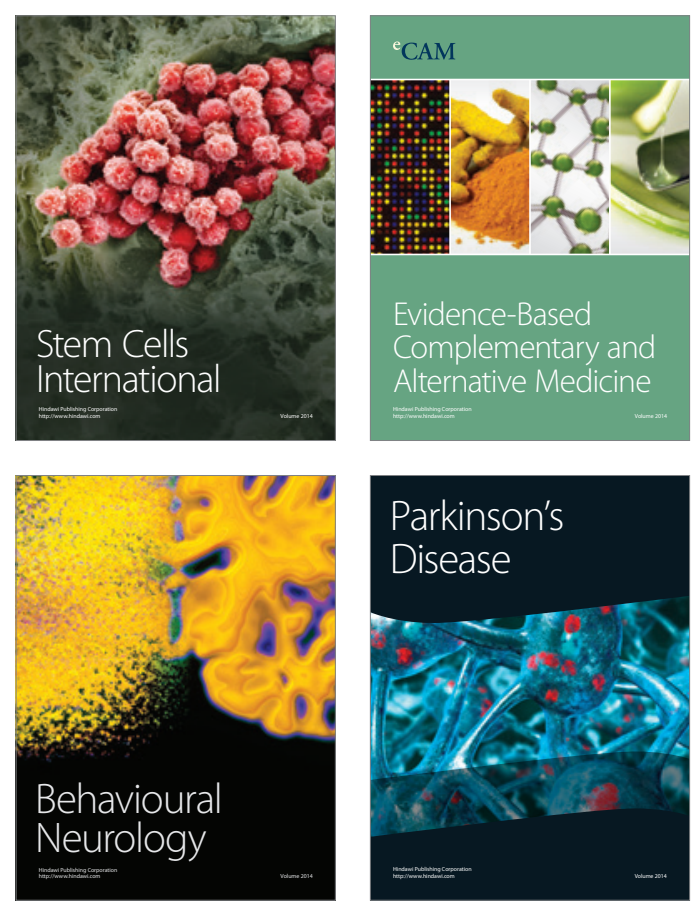

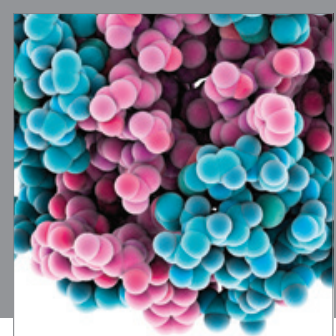

Journal of
Diabetes Research

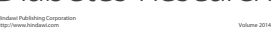

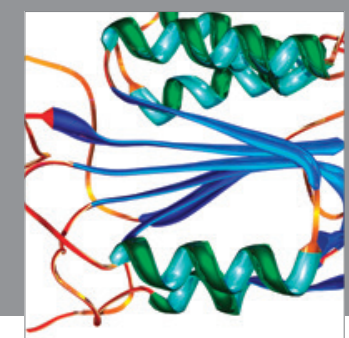

Disease Markers
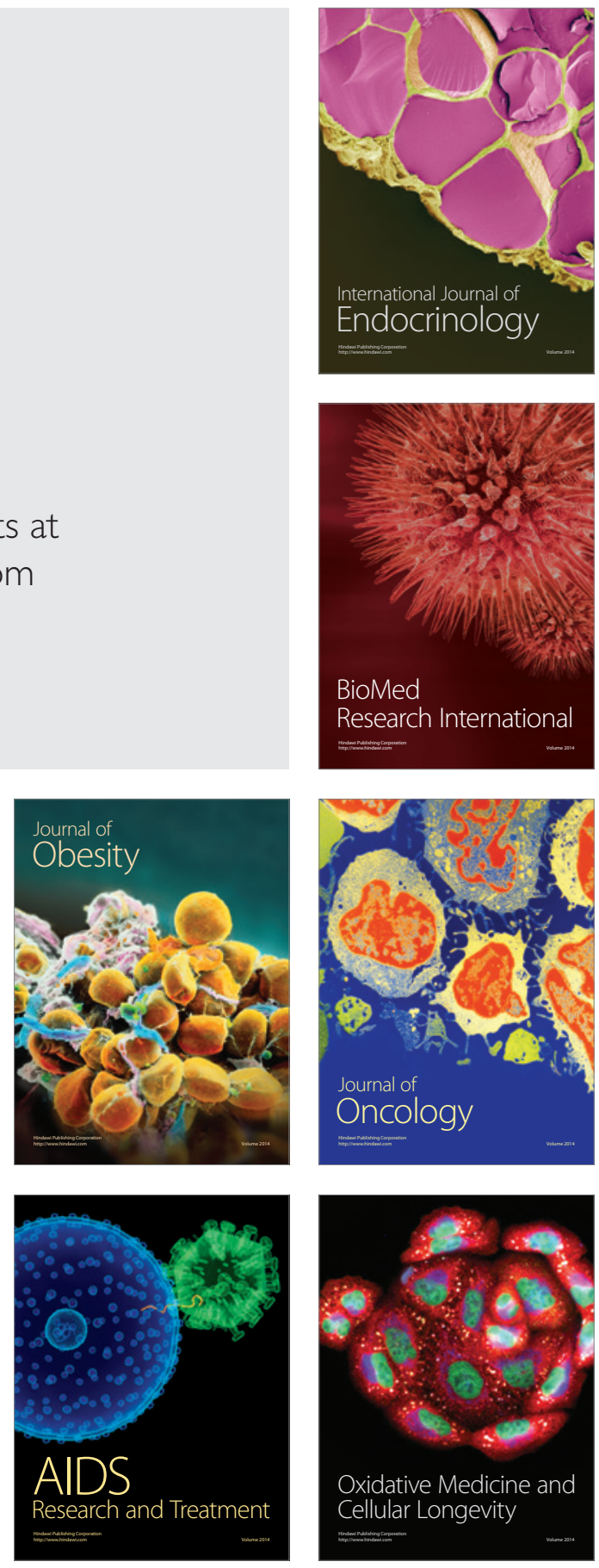